\title{
Piezoelectric Mimicry of Flexoelectricity
}

\author{
Amir Abdollahi* \\ Laboratori de Càlcul Numèric (LaCàN), Universitat Politècnica de Catalunya (UPC), \\ Campus Nord UPC-C2, E-08034 Barcelona, Catalonia, Spain \\ Fabián Vásquez-Sancho \\ Catalan Institute of Nanoscience and Nanotechnology (ICN2), CSIC \\ and The Barcelona Institute of Science and Technology, \\ Campus UAB, Bellaterra, 08193 Barcelona, Catalonia, Spain \\ and Centro de Investigación en Ciencia e Ingeniera de Materiales, \\ Universidad de Costa Rica, San José 11501, Costa Rica \\ Gustau Catalan ${ }^{\dagger}$ \\ Catalan Institute of Nanoscience and Nanotechnology (ICN2), CSIC \\ and The Barcelona Institute of Science and Technology, \\ Campus UAB, Bellaterra, 08193 Barcelona, Catalonia, Spain \\ and ICREA-Institut Catala de Recerca I Estudis Avançats, Barcelona, Catalonia, Spain
}

(Received 2 July 2018; published 15 November 2018)

\begin{abstract}
The origin of "giant" flexoelectricity, orders of magnitude larger than theoretically predicted, yet frequently observed, is under intense scrutiny. There is mounting evidence correlating giant flexoelectriclike effects with parasitic piezoelectricity, but it is not clear how piezoelectricity (polarization generated by strain) manages to imitate flexoelectricity (polarization generated by strain gradient) in typical beam-bending experiments, since in a bent beam the net strain is zero. In addition piezoelectricity changes sign under space inversion but giant flexoelectricity is insensitive to space inversion, seemingly contradicting a piezoelectric origin. Here we show that, if a piezoelectric material has its piezoelectric coefficient asymmetrically distributed across the sample, it will generate a nonzero bending-induced polarization impossible to distinguish from true flexoelectricity even by inverting the sample. The effective flexoelectric coefficient caused by piezoelectricity is functionally identical to, and often larger than, intrinsic flexoelectricity: our calculations show that, for standard perovskite ferroelectrics, even a tiny gradient of piezoelectricity $(1 \%$ variation of piezoelectric coefficient across $1 \mathrm{~mm}$ ) is sufficient to yield a giant effective flexoelectric coefficient of $1 \mu \mathrm{C} / \mathrm{m}$, three orders of magnitude larger than the intrinsic expectation value.
\end{abstract}

DOI: 10.1103/PhysRevLett.121.205502

Flexoelectricity is attracting growing attention due to its ability to replicate the electromechanical functionality of piezoelectric materials, which enables the use of lead-free dielectrics as flexoelectric replacements for piezoelectrics $[1,2]$. Experimental research on this phenomenon is still in relative infancy, but already there have been controversies about the magnitude, origin, and even thermodynamic reversibility of the flexoelectric effect [3-5]. Some of these controversies are starting to get settled, and, in particular, there is by now abundant evidence and growing consensus that seemingly "giant" flexoelectric effects are correlated with parasitic piezoelectric contributions from polar nanoregions [6], defect concentration gradients [7], residual ferroelectricity [8], or surfaces [9-12]. But, while the recent evidence suggests that indeed piezoelectricity can mimic flexoelectricity (which is the converse of flexoelectricity replicating piezoelectricity), it is not clear how (i.e., what are the necessary conditions for piezoelectricity to be able to imitate flexoelectricity), nor to what extent the "disguise" is perfect; i.e., can intrinsic flexoelectricity and flexoelectriclike piezoelectricity be experimentally distinguished?

To illustrate these questions, consider the following example: polarization can be generated by flexoelectricity when the applied deformation is inhomogeneous, e.g., when a sample is bent [13-18], but this is not necessarily true for piezoelectricity: bending a homogeneously poled piezoelectric beam will not elicit any piezoelectric polarization, see Fig. 1(a), because there is no net strain: the piezoelectric polarization caused by stretching on the convex side will be canceled by the opposite polarization caused by compression on the concave side. Symmetrywise, bending-induced piezoelectricity is precluded in a homogeneous material unless higher-order couplings are considered, and the deformations required for these are only achievable in 2D materials or near structural discontinuities [19-21]. 
(a)

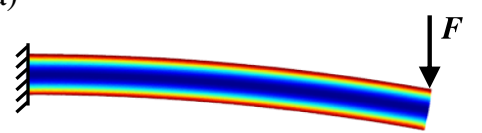

IV

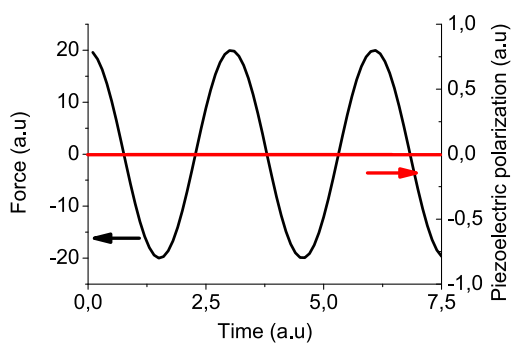

(b)
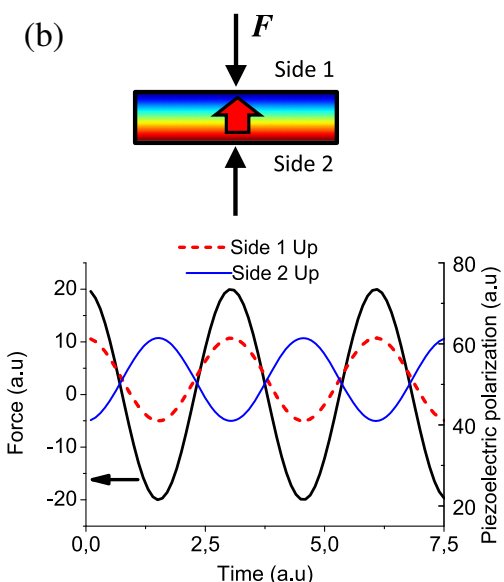

(c)


FIG. 1. (a) Homogeneously poled piezoelectric beam under bending, which does not induce a nonzero net polarization because the average strain is zero. The color plot presents the electric potential distribution. (b) Piezoelectric polarization induced in a rectangular sample under tension or compression. The red arrow represents the direction of the material polarization. (c) Flexoelectric polarization induced in a cantilever beam under bending. The polarization does not change sign by reversing the beam.

It follows from the previous discussion that the apparently giant flexoelectricity measured by bending some macroscopically polar materials [7] cannot be caused by a homogeneous piezoelectric state. Furthermore, because the existence of macroscopic piezoelectricity can be established by space-inversion experiments such as flipping the sample upside down and verifying that the sign of the stress-generated charge changes sign [7], see Fig. 1(b), it was assumed that such space-inversion tests could also be used to distinguish between piezoelectricity and flexoelectricity [10]. Indeed, the bending-induced polarization of a flexoelectric cantilever is independent of its orientation, see Fig. 1(c), but, as we will see, this inversion invariance can also hold for bent piezoelectric cantilevers.

Here, we analyze the electromechanical response of a bent piezoelectric beam, one of the common setups to quantify flexoelectricity [4], and show that (i) it is a necessary and sufficient condition that the piezoelectric coefficient be asymmetrically distributed for the beam to be able to replicate the functional behavior of a flexoelectric and (ii) that such asymmetric piezoelectricity cannot be distinguished from flexoelectricity in beambending experiments, even if the sample is turned upside down; the disguise is, in this respect, perfect. It is also possible to define an effective flexoelectric constant as a function of the spatial distribution of piezoelectricity. Quantitative analysis of this piezo-flexoelectric coefficient shows that even a relatively modest asymmetry in the distribution of piezoelectricity can lead to an effectively giant flexoelectric effect.

The constitutive equation for the electric displacement $\mathbf{D}$ in a linear dielectric solid possessing piezoelectricity and flexoelectricity is

$$
D_{i}=e_{i k l} \varepsilon_{k l}+\mu_{i j k l} \nabla_{l} \varepsilon_{j k}+\epsilon_{i j} E_{j}=\epsilon_{0} E_{i}+P_{i},
$$

where $\mathbf{E}$ is the electric field, $\varepsilon$ is the mechanical strain, $\mathbf{P}$ is the polarization, $\nabla \varepsilon$ is the strain gradient, $\mathbf{e}$ is the piezoelectric tensor, $\mu$ is the flexoelectric tensor, $\epsilon$ is the dielectric tensor, and $\epsilon_{0}$ is the permittivity of vacuum or air. We begin by analyzing the response of a piezoelectric flexoelectric cantilever beam under bending, see Fig. 2(a). We assume that the electric field and polarization exist only in the beam thickness direction $(z)$ since it has been shown that the longitudinal electric field is negligible compared with that in the beam thickness direction [22,23]. Then, Eq. (1) simplifies to

$$
D_{z}=e_{31} \varepsilon_{x x}+\mu_{13} \varepsilon_{x x, z}+\epsilon_{33} E_{z}=\epsilon_{0} E_{z}+P_{z},
$$

where the notation $e_{311}=e_{31}$ and $\mu_{1331}=\mu_{13}$ are introduced for convenience. Note that $\mu_{13}$, commonly reported in beam-bending experiments, is actually an effective flexoelectric coefficient involving a combination of flexoelectric tensor components $[4,16]$. In a bent beam, the strain components $\varepsilon_{y y}$ and $\varepsilon_{z z}$ are related to the strain component $\varepsilon_{x x}$ through the Poisson's ratio $\nu$ as $\varepsilon_{y y}=\varepsilon_{z z}=-\nu \varepsilon_{x x}$ [24]. The corresponding through-thickness strain gradients are then obtained as $\varepsilon_{y y, z}=\varepsilon_{z z, z}=-\nu \varepsilon_{x x, z}$. This relationship is encoded in the effective flexoelectric coefficient as $\mu_{13}=\bar{\mu}_{13}-\nu\left(\bar{\mu}_{13}+\bar{\mu}_{11}\right)$, where $\bar{\mu}_{11}$ and $\bar{\mu}_{13}$ are the longitudinal and transverse components of the flexoelectric tensor, respectively. The same applies to the effective piezoelectric coefficient $e_{31}=\bar{e}_{31}-\nu\left(\bar{e}_{31}+\bar{e}_{33}\right)$, where $\bar{e}_{33}$ and $\bar{e}_{31}$ are the longitudinal and transverse components of the piezoelectric tensor, respectively.

In the absence of surface charges and applied voltage, the electrostatic equilibrium (Maxwell' s equation) leads to

$$
D_{z}=\epsilon_{0} E_{z}+P_{z}=0 \text {. }
$$

Plugging this equation in Eq. (2) and using the Euler beam hypotheses $\varepsilon_{x x}=-\kappa z$ and $\varepsilon_{x x, z}=-\kappa$, where $\kappa$ is 


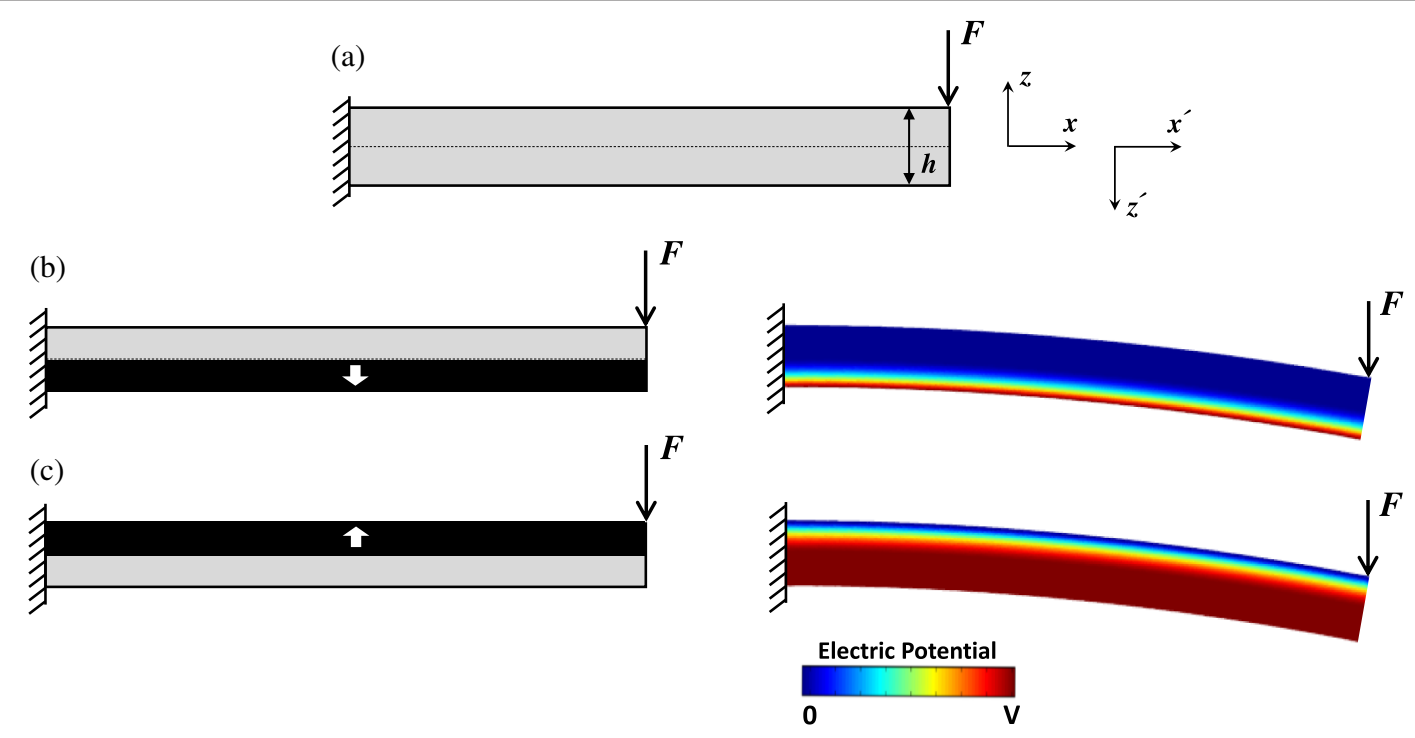

FIG. 2. (a) Schematic of a cantilever beam under the point load $F$. (b) Schematic of a bimorph cantilever beam where the dark layer is piezoelectric. (c) A reversed configuration of the bimorph (rotated by $180^{\circ}$ ). The white arrows represent the polarization direction of the layers. The color plot presents the electric potential $(\phi)$ distribution for each bimorph obtained from Eqs. (3) and (4), where $E_{z}=-\phi_{, z}$. The net polarization can be obtained from the electric potential difference of the top and bottom faces, which is identical in both bimorphs.

the beam curvature induced by the applied force $F$, the polarization in the $z$ direction can be obtained as

$$
P_{z}(z)=-\frac{\kappa}{\epsilon_{r}}\left(e_{31} z+\mu_{13}\right),
$$

where $\epsilon_{r}=\epsilon_{33} / \epsilon_{0}$ is the relative dielectric constant. We note that this equation can also be derived from analytical solutions of the electroelastic fields in bending piezoelectric cantilever beams with the flexoelectric effect $[17,25,26]$ or gradient piezoelectricity $[27,28]$. The total net polarization over the beam thickness is then obtained as

$$
P_{t}=\frac{1}{h} \int_{-h / 2}^{h / 2} P_{z} d z=-\frac{\kappa}{h \epsilon_{r}} \int_{-h / 2}^{h / 2}\left(e_{31} z+\mu_{13}\right) d z .
$$

In the absence of piezoelectricity, i.e., $e_{31}=0$, the polarization is only induced by the flexoelectric effect, resulting in a net polarization $P_{t}=-\mu_{13} \kappa / \epsilon_{r}$, independent of the beam direction. In other words, the net polarization induced by flexoelectricity does not change sign by reversing the beam, as expected.

We can also use Eq. (5) to obtain the net polarization corresponding to a beam where the electromechanical response is piezoelectric instead of flexoelectric, i.e., $\mu_{13}=0$. However, bending a homogeneous piezoelectric beam is unable to produce a nonzero net polarization since $e_{31} z$ is antisymmetric about the center of the beam $(z=0)$, thus leading to a zero net polarization in Eq. (5); see Fig. 1(a). The physical reason, as mentioned at the introduction, is that opposite stresses (compressive and tensile) are induced in the upper and lower halves of the beam, respectively, resulting in opposite piezoelectric effects and a zero net polarization. Therefore, bending a homogeneous piezoelectric beam cannot give a flexoelectriclike response.

One way to break the balance of charges in a bent piezoelectric beam is to replace the top or bottom layers with a different piezoelectric or even a nonpiezoelectric layer, as is done, for example, in piezoelectric bimorph sensors and actuators [29,30]. One such bimorph is illustrated in Fig. 2(b), in which the bottom layer is piezoelectric while the top layer is not, i.e., $e_{31}=0$ for $h \geq 0$ and $-e_{31}$ for $h<0$. The bimorph can be seen as an extreme case of asymmetric piezoelectricity, where $e_{31}$ is a Heaviside step function. In this case the net polarization is obtained from Eq. (5) as $P_{t}=-e_{31} h \kappa / 8 \epsilon_{r}$.

A bimorph piezoelectric cantilever thus generates a polarization just like a flexoelectric cantilever would. Moreover, the sign (phase shift) of the piezoelectric polarization does not change by reversing the beam. Figure 2(c) presents the reversed configuration which is equivalent to consider $e_{31}=0$ for $h<0$. Plugging these conditions in Eq. (5) leads to a net polarization $P_{t}=$ $-e_{31} h \kappa / 8 \epsilon_{r}$, identical both in magnitude and sign, to the induced polarization in the original bimorph in Fig. 2(b). Therefore, a bent piezoelectric bimorph is qualitatively indistinguishable from a bent flexoelectric beam.

We can generalize the conclusions of this example. Let us assume a generic cantilever beam with an arbitrary distribution of piezoelectricity $e_{31}(z)$. Equation (5) results in a zero net polarization if the piezoelectricity is symmetrically distributed about the centre of the beam, i.e., for any $e_{31}(z)$ such that $e_{31}(z)=e_{31}(-z)$. Mathematically the 
integrand is antisymmetric about the centre of the beam for any such symmetrical distribution of piezoelectricity. Conversely, any asymmetry in the distribution of piezoelectricity such that $e_{31}(z) \neq e_{31}(-z)$ will result in a nonzero integral and thus in a net bending-induced piezoelectric polarization.

In addition, the sign of the net polarization in Eq. (5) does not change by flipping the beam. In the flipped configuration, the coordinate system $x-z$ converts to the new system $x^{\prime}-z^{\prime}$, where $z^{\prime}=-z$. Using this conversion and taking into account the negative sign of $e_{31}$ in the flipped configuration, Eq. (5) converts to an identical equation as a function of $z^{\prime}$, retaining its sign. Therefore, for a piezoelectric beam to be able to indistinguishably mimic a flexoelectricity [i.e., for Eq. (5) to yield a nonzero solution that is invariant with respect to space inversion], it is necessary and sufficient that the piezoelectric coefficient be asymmetrically distributed across the thickness of the beam. An example of this general concept is, as mentioned, the bimorph piezoelectric cantilever, for which $e_{31}(z)$ is a step function. Another example is surface piezoelectricity, an inevitable element of the total flexoelectric response $[4,10,12,31,32]$, which can be modeled as two antisymmetric step functions in $e_{31}(z)$, see the Supplemental Material [33].

Since asymmetric piezoelectricity can mimic flexoelectricity, it is possible to define an effective flexoelectric constant as a function of the distribution of piezoelectricity. For a flexoelectric cantilever, the induced polarization as a function of the beam curvature is given by $P_{t}=-\mu_{e} \kappa / \epsilon_{r}$, where $\mu_{e}$ is the effective flexoelectric constant. By equating this polarization to Eq. (5) with $\mu_{13}=0$, the effective flexoelectric constant becomes

$$
\mu_{e} \equiv \frac{1}{h} \int_{-h / 2}^{h / 2} e_{31}(z) z d z .
$$

In order to get some quantitative estimates of how much pseudoflexoelectricity we can elicit from a gradient of piezoelectricity, we consider a simple linear distribution of piezoelectricity as $e_{31}(z)=z \Delta e / h+e_{0}$, where $\Delta e / h$ is the slope of the linear gradient of piezoelectricity and $e_{0}=e_{31}(0)$. Plugging this function into Eq. (6) yields an effective flexoelectric coefficient of

$$
\mu_{e}=h \Delta e / 12 .
$$

Let us use this equation to analyze relevant experimental cases. Experimental setups to quantify flexoelectricity commonly employ cantilever beams with a thickness in the order of $h=1 \mathrm{~mm}[14,15,34-37]$. Therefore, to induce a typical "giant" flexoelectric coefficient on the order of $\mu_{e}=1 \mu \mathrm{C} / \mathrm{m}$, as reported for important piezoelectric materials such as PZT and $\mathrm{BaTiO}_{3}[14,15,36]$, the piezoelectric variation $\Delta e$ between the two sides of the $1 \mathrm{~mm}$ sample should be of the order of $10^{-2} \mathrm{C} / \mathrm{m}^{2}$. Compared to the average piezoelectric coefficient of $\mathrm{PZT}$ and $\mathrm{BaTiO}_{3}$, which is on the order of $5 \mathrm{C} / \mathrm{m}^{2}[38,39]$, this gradient is equivalent to a $0.2 \%$ change of the piezoelectric constant across the beam thickness. Therefore, for materials with big piezoelectric coefficients, even such a tiny gradient of piezoelectricity can yield an apparently giant flexoelectricity. This calls into question bending-based quantifications of flexoelectricity in the polar phase of ferroelectrics and highlights the need for alternative experimental approaches.

Another relevant question, of course, is to what extent these results can be extended to nominally paraelectric materials. As has recently been reported, even in a theoretically paraelectric phase, a gradient of defects can result in a small but measurable macroscopic piezoelectricity [7]. The reported effective piezoelectric coefficients for paraelectric perovskites is in the order of $0.05 \mathrm{C} / \mathrm{m}^{2}$. With this average value, the piezoelectric gradient of $10^{-2} \mathrm{C} / \mathrm{m}^{2}$ across $1 \mathrm{~mm}$ needed to yield $\mu_{e}=1 \mu \mathrm{C} / \mathrm{m}$ requires a 20\% variation of piezoelectricity across the $1 \mathrm{~mm}$ thick beam. Though this gradient is large, it is not unfeasible. Parenthetically, we note that thermal gradients can cause differences in thermal expansion and thus also gradient-induced polarization [40,41]. Temperature differences between electrodes may therefore also cause paraelectric materials to behave like piezoelectrics.

Based on the present analysis, we can also suggest the following approaches to separate asymmetric piezoelectricity from true flexoelectricity:

i. Piezoelectric gradients are anisotropic. $\Delta e$ in Eq. (7) is maximum in the direction of the gradient but zero perpendicular to it. One can therefore perform beambending experiments with different sample cuts to change the orientation of asymmetric piezoelectricity with respect to the through-thickness strain gradient. In single crystals there can also be inherent crystal anisotropy in addition to extrinsic gradients, but in ceramics the random orientation of crystallites implies that any measured anisotropy must be due to extrinsic gradients.

ii. Equation (7) also indicates that the piezoelectric contribution is proportional to sample size. We can therefore perform beam-bending experiments with different sample thicknesses.

iii. The size dependency also suggests performing local measurements. Since the more localized the measurement, the smaller the contribution from spatial distributions, a truly local measurement would be immune to any asymmetric distribution of piezoelectricity. The more local the measurement, the smaller the contribution from spatial differences of piezoelectricity. In some mechanical measurements, such as indentation or fracture experiments, strain gradients are generated in extremely small volumes [42-44], and therefore flexoelectric measurements based on nanomechanical measurements or fracture mechanics will be generally more robust against asymmetric piezoelectricity. 
In summary, we show that piezoelectricity can indeed imitate flexoelectricity (bending-induced polarization) on the condition that the piezoelectric coefficient is inhomogeneously and asymmetrically distributed across the sample. If this condition is met, asymmetric piezoelectricity becomes indistinguishable from intrinsic flexoelectricity in single cantilever-bending experiments. This mimicry complicates the task of interpreting experimental results although we have suggested some approaches to separate inhomogeneous piezoelectricity from flexoelectricity. Piezoelectric mimicry also represents a practical opportunity; just like flexoelectricity was initially conceived as a way for replicating the device functionality of piezoelectrics $[1,45,46]$, asymmetric piezoelectricity may also be used to imitate the interesting novel functionalities $[43,47-$ 49] provided by flexoelectricity.

This research was funded by an ERC Starting grant (ERC 308023), by the Severo Ochoa Excellence Programme (SEV-2013-0295), by MINECO Project No. MAT2016-77100-C2-1-P, and by the CERCA Programme from the Generalitat de Catalunya. F. V.-S. thanks UCR, MICITT and CONICIT for support.

*amir.abdollahi@upc.edu † gustau.catalan@icn2.cat

[1] B. Chu, W. Zhu, N. Li, and L. E. Cross, J. Appl. Phys. 106, 104109 (2009).

[2] U. K. Bhaskar, N. Banerjee, A. Abdollahi, Z. Wang, D. G. Schlom, G. Rijnders, and G. Catalan, Nat. Nanotechnol. 11, 263 (2016).

[3] P. V. Yudin and A. K. Tagantsev, Nanotechnology 24, 432001 (2013).

[4] P. Zubko, G. Catalan, and A. K. Tagantsev, Annu. Rev. Mater. Res. 43, 387 (2013).

[5] T. D. Nguyen, S. Mao, Y.-W. Yeh, P. K. Purohit, and M. C. McAlpine, Adv. Mater. 25, 946 (2013).

[6] J. Narvaez and G. Catalan, Appl. Phys. Lett. 104, 162903 (2014).

[7] A. Biancoli, C. Fancher, J. Jones, and D. Damjanovic, Nat. Mater. 14, 224 (2015).

[8] L. M. Garten and S. Trolier-McKinstry, J. Appl. Phys. 117, 094102 (2015).

[9] A. K. Tagantsev, Phys. Rev. B 34, 5883 (1986).

[10] J. Narvaez, S. Saremi, J. Hong, M. Stengel, and G. Catalan, Phys. Rev. Lett. 115, 037601 (2015).

[11] J. Narvaez, F. Vasquez-Sancho, and G. Catalan, Nature (London) 538, 219 (2016).

[12] X. Zhang, Q. Pan, D. Tian, W. Zhou, P. Chen, H. Zhang, and B. Chu, Phys. Rev. Lett. 121, 057602 (2018).

[13] E. V. Bursian and O. I. Zaikovskii, Sov. Phys. Solid State 10, 1121 (1968).

[14] W. Ma and L. E. Cross, Appl. Phys. Lett. 86, 072905 (2005).

[15] L. E. Cross, J. Mater. Sci. 41, 53 (2006).

[16] P. Zubko, G. Catalan, P. R. L. Welche, A. Buckley, and J. F. Scott, Phys. Rev. Lett. 99, 167601 (2007).
[17] M. S. Majdoub, P. Sharma, and T. Cagin, Phys. Rev. B 77, 125424 (2008).

[18] S. Kwon, W. Huang, L. Shu, F.-G. Yuan, J.-P. Maria, and X. Jiang, Appl. Phys. Lett. 105, 142904 (2014).

[19] I. Naumov, A. M. Bratkovsky, and V. Ranjan, Phys. Rev. Lett. 102, 217601 (2009).

[20] K. H. Michel, M. Neek-Amal, and F. M. Peeters, Phys. Status Solidi B 253, 2311 (2016).

[21] K. Chu and C.-H. Yang, Phys. Rev. B 96, 104102 (2017).

[22] Y. Gao and Z. L. Wang, Nano Lett. 7, 2499 (2007).

[23] G.-F. Wang and X.-Q. Feng, Europhys. Lett. Assoc. 91, 56007 (2010).

[24] L. D. Landau and E. M. Lifshitz, Theory of Elasticity, 3rd ed. (Pergamon, New York, 1986).

[25] M. S. Majdoub, P. Sharma, and T. Cagin, Phys. Rev. B 79, 119904(E) (2009).

[26] Z. Yan and L. Y. Jiang, J. Appl. Phys. 113, 194102 (2013).

[27] W. S. Williams, Ferroelectrics 41, 225 (1982).

[28] W. S. Williams, Ferroelectrics 51, 61 (1983).

[29] J. G. Smits, S. I. Dalke, and T. K. Cooney, Sens. Actuators A 28, 41 (1991).

[30] J. Pritchard, C. R. Bowen, and F. Lowrie, British Ceramic Transactions 100, 265 (2001).

[31] A. K. Tagantsev and A. S. Yurkov, J. Appl. Phys. 112, 044103 (2012).

[32] M. Stengel, Phys. Rev. B 90, 201112 (2014).

[33] See Supplemental Material at http://link.aps.org/ supplemental/10.1103/PhysRevLett.121.205502 for derivation of the surface-piezoelectric contribution to flexoelectricity starting from Eq. (5).

[34] W. Ma and L. E. Cross, Appl. Phys. Lett. 79, 4420 (2001).

[35] W. Ma and L.E. Cross, Appl. Phys. Lett. 81, 3440 (2002).

[36] W. Ma and L. E. Cross, Appl. Phys. Lett. 88, 232902 (2006).

[37] L. Shu, X. Wei, L. Jin, Y. Li, H. Wang, and X. Yao, Appl. Phys. Lett. 102, 152904 (2013).

[38] Z. Li, S. K. Chan, M. H. Grimsditch, and E. S. Zouboulis, J. Appl. Phys. 70, 7327 (1991).

[39] S. Zhu, B. Jiang, and W. Cao, Proc. SPIE Int. Soc. Opt. Eng. 3341, 154 (1998).

[40] V. A. Trepakov, K. M. Nurieva, and A. K. Tagantsev, Ferroelectrics 94, 377 (1989).

[41] A. K. Tagantsev, Phase Transitions 35, 119 (1991).

[42] A. Abdollahi, C. Peco, D. Millán, M. Arroyo, G. Catalan, and I. Arias, Phys. Rev. B 92, 094101 (2015).

[43] K. Cordero-Edwards, N. Domingo, A. Abdollahi, J. Sort, and G. Catalan, Adv. Mater. 29, 1702210 (2017).

[44] F. Vasquez-Sancho, A. Abdollahi, D. Damjanovic, and G. Catalan, Adv. Mater. 30, 1705316 (2018).

[45] W. Zhu, J. Y. Fu, N. Li, and L. Cross, Appl. Phys. Lett. 89, 192904 (2006).

[46] J. Y. Fu, W. Zhu, N. Li, N. B. Smith, and L. E. Cross, Appl. Phys. Lett. 91, 182910 (2007).

[47] H. Lu, C. W. Bark, D. E. D. L. Ojos, J. Alcala, C. B. Eom, G. Catalan, and A. Gruverman, Science 336, 59 (2012).

[48] A. S. Starkov and I. A. Starkov, Int. J. Solids Struct. 82, 65 (2016).

[49] M.-M. Yang, D. J. Kim, and M. Alexe, Science 362, 3256 (2018). 